\title{
Introduction to the Special Issue on ASSETS'17 (Part 2)
}

This issue is a continuation of the Special Issue on ASSETS'17, which includes articles that are extended versions of conference papers presented at the 19th International ACM SIGACCESS Conference on Computers and Accessibility (ASSETS'17). Authors of top papers from the conference submitted manuscripts, which underwent a full review process for ACM Transactions on Accessible Computing (TACCESS). The guest editor for this issue is Anke M. Brock (ENAC, Université Toulouse, France), and Kathleen F. McCoy (University of Delaware) served as the primary editorin-chief, to ensure that conflicts of interest were carefully managed throughout the review process.

The first three articles of this special issue appeared in an earlier issue of TACCESS (Volume 12 Issue 2), and we now present a fourth article that has been accepted to this special issue. Once again, we thank the authors for their high-quality submissions, and we also thank the reviewers who have contributed their time and expertise to this process.

The paper included in this second part of the special issue, "NavCog3 in the Wild: LargeScale Blind Indoor Navigation Assistant with Semantic Features," presents and evaluates a system for turn-by-turn navigation assistance to enable independent navigation in indoor environments by people with visual impairments.

-Matt Huenerfauth and Kathleen F. McCoy Editors-in-Chief 